\title{
Calibrating Resistance with Wheatstone Bridge and SPSS
}

\author{
Qing YE, Xinchun WANG, Kaihua YUE \& Man CHENG \\ School of Physics and Electronics, Chuxiong Normal University, Chuxiong 675000, China
}

\begin{abstract}
Based on the balance principle of Wheatstone Bridge, this paper firstly derives a linear relationship between comprehensive quantity (of resistance \& length) and the length of resistance wire, then measures experimental data of resistance and length, finally introduces SPSS to analyze data, verify the linear relationship between comprehensive quantity and length, and determine the resistance to be measured. In this paper, uncertainty estimation (confidence probability of 95\%) is used to evaluate experimental data and results. All these makes the final results more reasonable.
\end{abstract}

KEYWORD: Wheatstone Bridge; Balance principle; Uncertainty analysis; Linear estimation of SPSS; Calibrating resistance

\section{INTRODUCTION}

Wheatstone Bridge, a DC Bridge using electric potential comparative method, is widely used in electric measurement technology and automatic control measurement because of its high sensitivity and accuracy[7]. Investigating the use and characteristics of DC Bridge (Wheatstone Bridge)[8] is an important experiment of college physics. According to reference [1][3][9]we know that, DC Bridge is suitable for measuring resistance among 10 to $10^{6} \Omega$. Measuring resistance with DC Bridge is based on comparative method. In this paper, uncertainty estimation (confidence probability of $95 \%$ ) is used to evaluate experimental data. In addition, by use of the curve estimation function of SPSS[6], error caused by instrument or human factors is reduced significantly. All these makes the experimental results more reasonable.

\section{EXPERIMENTAL INSTALLATION AND ADJUSTING}

The experimental installation of Wheatstone Bridge shown in Figure 1 is mainly composed of a regulated power supply, two sliding rheostats, a resistance box, a galvanometer and a switch. The resistance of a resistor is to be measured.

In Figure 1, turn off the switch, adjust the galvanometer pointer to zero line, adjust the resistance box to be zero, adjust the two sliding rheostats to achieve the function of protection circuit, and fix the standard resistor to be $2400 \Omega$.

Figure 1. Experimental installation of Wheatstone Bridge

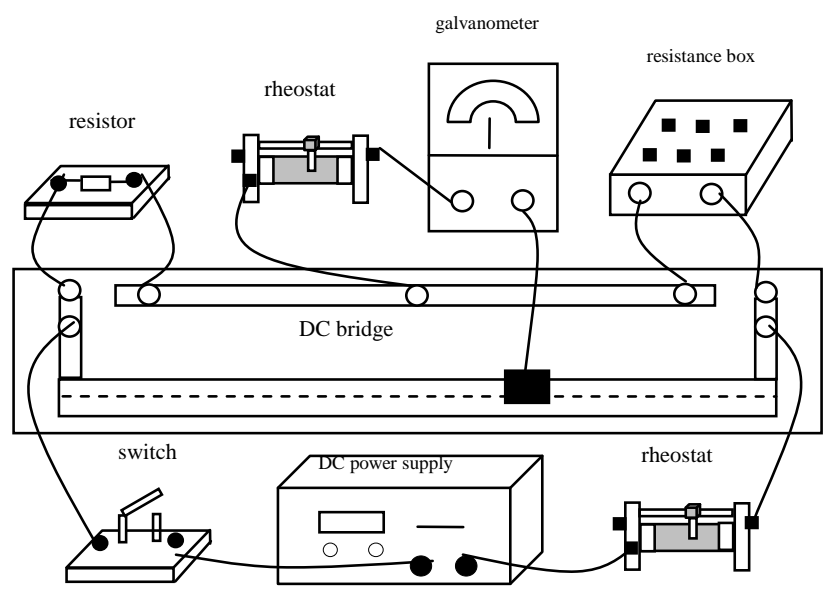

Turn on the switch; move the Wheatstone Bridge's sliding contact and get the left length $L_{1}$ and the right length $L_{2}$ of the resistance wire (both relative to the contact point). Then adjust the resistance box until the galvanometer pointer to zero line again. Record $R_{S}$ from the resistance box, and read $L_{1}$ and $L_{2}$ from the meter ruler. 


\section{EXPERIMENTAL PRINCIPLE}

\subsection{Measurement principle}

The experimental circuit is shown in Figure 2. If the Bridge is not in balance, there is a current through the galvanometer, so the galvanometer pointer deflects. Adjust $R_{S}$ to a proper value, the two electric potentials of nodes $\mathrm{C}$ and $\mathrm{D}$ equals, and current through the galvanometer becomes $I_{g}=0$, then the Bridge is in balance.

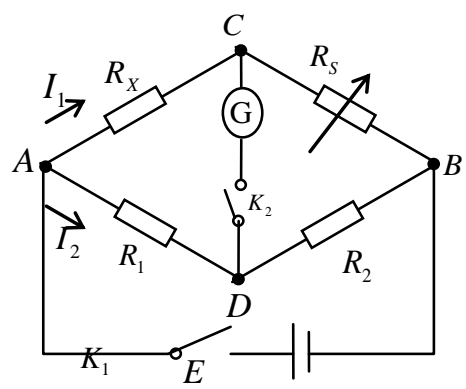

Figure 2. Experimental circuit

Therefore

$U_{A C}=U_{A D}=I_{1} R_{X}=I_{2} R_{1}$

$U_{C D}=U_{D B}=I_{1} R_{S}=I_{2} R_{2}$

Making use of equation (1) and equation (2) gives

$R_{X}=\frac{R_{1}}{R_{2}} R_{S}$

In experiment, $R_{1}$ and $R_{2}$ are instead by a uniform resistance wire. By the definition of resistance [5] we have

$R_{1}=\rho \frac{L_{1}}{S}$

$R_{2}=\rho \frac{L_{2}}{S}$

Substituting equation (4) and equation (5) into (3) gives

$R_{X}=\frac{L_{1}}{L_{2}} R_{S}$

Let $Y=L_{1} R_{S}$ to be a comprehensive quantity of resistance and length, then equation (6) can be simplified as

$Y=R_{X} L_{2}$

In the experiment, the DC Bridge is always kept on balance by adjusting $R_{S}$ and changing sliding contact (therefore changing $L_{1}$ and $L_{2}$ ). Measure $L_{1}$ and $L_{2}$ with a ruler, and read $R_{S}$ from the resistance box, so we get comprehensive quantity
$Y_{i}$ of resistance and length. By use of the linear estimation function of SPSS, analyze the linear correlation between comprehensive quantity $Y_{i}$ and length $L_{2 i}$, then calibrate the resistance $R_{X}$ and estimate its uncertainty.

\subsection{Uncertainty analysis of measuring resistance with DC Bridge}

For a direct measurement quantity $r$, its uncertainty can be divided into two classes, namely class A and class B. For a series of measurements, the standard deviation of average value is [4]

$u_{(\bar{r})}=\sqrt{\frac{\sum_{i=1}^{n}\left(r_{i}-\bar{r}\right)^{2}}{n(n-1)}}$

In class $A$, when the measuring number is 8 , the measurement results obeys $t$ distribution [4]; when $p=0.95$, then $t_{p}=2.36$, that is,

$u_{A(\bar{r})}=2.36 u_{(\bar{r})}$

where $\bar{r}$ in equation (9) stands for $\bar{L}_{1}, \bar{L}_{2}$ or $\bar{R}_{S}$ respectively.

In class $B$, the instrument limit error $\Delta$ is subject to uniform distribution [4] $C=\sqrt{3}$; when $p=0.95$, then $k_{p}=1.96$, therefore,

$u_{B(r)}=1.96 \frac{\Delta_{r}}{\sqrt{3}}$

where $r$ in equation (10) stands for $L_{1}, L_{2}$ or $R_{S}$ respectively.

The uncertainty of a direct measurement quantity $r$ is

$u_{(r)}=\sqrt{u_{A(\bar{r})}^{2}+u_{B(r)}^{2}}$

For an indirect measurement quantity $y=f\left(r_{1}, r_{2}, \cdots, r_{n}\right)$, its standard uncertainty is $u_{c(y)}$ [2].

$u_{c(y)}=\sqrt{\sum_{i=1}^{n}\left(\frac{\partial y}{\partial r}\right)^{2} u^{2}{ }_{\left(r_{i}\right)}}$

The relative uncertainty of $y$ is $u_{r(y)}$ :

$u_{r(y)}=\sqrt{\sum_{i=1}^{n}\left(\frac{\partial(\ln y)}{\partial r_{i}}\right)^{2} u_{\left(r_{i}\right)}^{2}}$

Considering equations (7) and (13), the relative uncertainty of the measured resistance $R_{X}$ is

$u_{r\left(R_{X}\right)}=\sqrt{\left(\frac{u_{(Y)}}{Y}\right)^{2}+\left(\frac{u_{\left(L_{2}\right)}}{L_{2}}\right)^{2}}$ 


\section{EXPERIMENT DATA PROCESSING}

\subsection{Experimental results and data processing}

Table1. Measurement value of $R_{S}$ when contact in different position

\begin{tabular}{|c|c|c|c|}
\hline$i$ / Group & $L_{1 i} / \mathrm{cm}$ & $L_{2 i} / \mathrm{cm}$ & $R_{S i} / \mathrm{k} \Omega$ \\
\hline 1 & 70.00 & 30.00 & 1.03140 \\
\hline 2 & 65.00 & 35.00 & 1.29360 \\
\hline 3 & 60.00 & 40.00 & 1.59840 \\
\hline 4 & 55.00 & 45.00 & 1.97430 \\
\hline 5 & 50.00 & 50.00 & 2.41120 \\
\hline 6 & 45.00 & 55.00 & 2.94440 \\
\hline 7 & 40.00 & 60.00 & 3.60520 \\
\hline 8 & 35.00 & 65.00 & 4.45530 \\
\hline
\end{tabular}

where $R=2400.00 \Omega$ (the resistor to be measured), $\Delta_{R}=0.1 \Omega$ and $\Delta_{L}=0.1 \mathrm{~cm}$.

Table2. The experimental data of comprehensive quantity $Y_{i}$ vs. length $L_{2 i}$

\begin{tabular}{|c|c|c|}
\hline$i /$ Group & $Y_{i} / 10^{4} \Omega \cdot \mathrm{cm}$ & $L_{2 i} / 10 \mathrm{~cm}$ \\
\hline 1 & 7.219800 & 3.000 \\
\hline 2 & 8.408400 & 3.500 \\
\hline 3 & 9.590400 & 4.000 \\
\hline 4 & 10.858650 & 4.500 \\
\hline 5 & 12.056000 & 5.000 \\
\hline 6 & 13.249800 & 5.500 \\
\hline 7 & 14.420800 & 6.000 \\
\hline 8 & 15.593550 & 6.500 \\
\hline
\end{tabular}

\subsection{Using SPSS software to analysis calibration curve of $Y-L_{2}$}

Input experimental data in Table 2 to SPSS software, taking $L_{2}$ as variable and $Y$ as dependent variable. Apply the function of curve estimation of SPSS, we have the calibration curve equation:

$$
Y=2.401280952380952 \times 10^{3} L_{2}
$$

$$
+0.01859047619047976
$$

The calibration curve is shown in Figure 3.

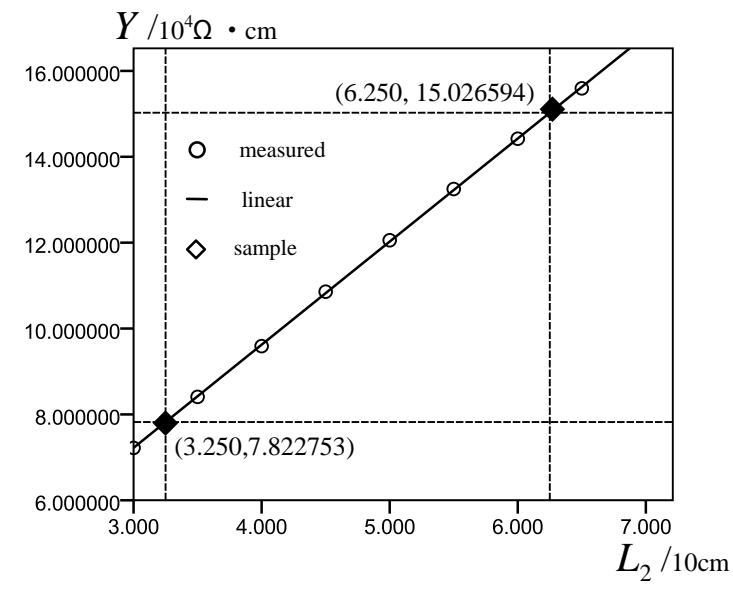

Figure 3. $Y-L_{2}$ Calibration curve obtained by SPSS

\subsection{Estimation of uncertainty of measured resistance}

According to the calibration curve $Y-L_{2}$ (straight line) in figure 3, take two sampling points $\left(L_{31}, Y_{1}\right)$ and $\left(L_{22}, Y_{2}\right)$ from the straight line, we have

$R_{X}=\frac{Y_{2}-Y_{1}}{L_{22}-L_{21}}$

By equation (15) we get

$u_{\left(Y_{j}\right)}=2.401280952380952 \times 10^{3} L_{2 j}$

By equation (16) and equation (17) we get

$u_{r\left(R_{X}\right)}=\frac{u_{\left(R_{X}\right)}}{R_{X}}=\sqrt{\left(\frac{u_{\left(Y_{2}-Y_{1}\right)}}{Y_{2}-Y_{1}}\right)^{2}+\left(\frac{u_{\left(L_{22}-L_{2}\right)}}{L_{2_{1}}-L_{21}}\right)^{2}}$

From the two sampling points $\left(L_{21}, Y_{1}\right)$ and $\left(L_{22}, Y_{2}\right)$ in Figure 3, combined with equations (16), (17) and (18) can get the Table 3 as the final experimental results.

Table 3. Experimental results of DC Bridge for measuring resistance

\begin{tabular}{|c|c|c|c|}
\hline $\begin{array}{c}u_{\left(Y_{1}\right)}=u_{\left(Y_{2}\right)} \\
/ \Omega \cdot \mathrm{cm}\end{array}$ & $\begin{array}{c}u_{\left(L_{22}\right)}=u_{\left(L_{21}\right)} \\
/ \mathrm{cm}\end{array}$ & $\begin{array}{c}R_{X} \\
/ 10^{3} \Omega\end{array}$ & $u_{r\left(R_{X}\right)}$ \\
\hline 0.27 & 0.11 & $2.401 \pm 0.018$ & $0.75 \%$ \\
\hline
\end{tabular}

\section{ANALYSIS AND DISCUSSION}

From equation (7) we know that, as long as the measurement conditions been satisfied, a linear relationship exists between comprehensive quantity $Y$ ( of resistance and length) and length $L_{2}$. If the linear relationship is obtained from experiment, the value of the resistance of the resistor can be calibrated consequentially. This method of 
measuring resistance has certain innovation in experimental principle.

In order to make experimental data more rational for analysis, the total length $\left(L_{1}+L_{2}\right)$ is kept at $100 \mathrm{~cm}$, and the step length of $L_{1}$ and $L_{2}$ is $5 \mathrm{~cm}$. These selections make the resistance box $R_{S}$ to change from $1 \mathrm{k} \Omega$ to $4.5 \mathrm{k} \Omega$, which thus can make the measurement results more reliable and reasonable.

From experimental data in Table2, by means of the linear estimation function of SPSS, we get calibration curve equation (15) and calibration curve $Y-L_{2}$. Obviously, comprehensive quantity $\left(Y_{i}\right)$ and length $\left(L_{2 i}\right)$ have linear relationship (see Figure3). All these are consistent to theoretical equation (7).

From the experimental results in Table 3, we know the measured value of the resistor is $2.401 \times 10^{3} \Omega$. Comparing with the calibration resistor of $2.400 \times 10^{3} \Omega$, the two values are consistent each other, which indicates that the experimental fitting line $Y-L_{2}$ is objective. Also, the experimental results indicate that, by use of the curve estimation function of SPSS, the procedure of the data processing becomes simple and clear, and analysis accuracy of experimental data and experimental results is improved significantly.
In brief, the experiment scheme and the method in this paper are worth adopting.

\section{REFERENCES}

[1] Kang Yurong \& Kang Xiangdong. 2004. Discussion in DC resistance measurement to improve the accuracy of single Bridge. Journal of Xi'an University of science and technology. vol. 24 No. 3.

[2] Liu Caiming. University physics experiment in the measurement uncertainty

[3] Lv Guangming. 2013. High precision measurement method for resistance by DC Bridge. Quality and technical supervision. No. 2.

[4] Liu Jianwei \& Wang Xinchun. 2013. The calibration of acceleration of gravity with improved single pendulum experiment system and SPSS. University physics experiment. vol. 26. No.4: $56-57$.

[5] Ma Junjie. 2006. Explore the law of resistance with digital multimeter. Explore in physical science. vol. 21.

[6] Song Zhigang. 2008. SPSS 16.0 Guide to data analysis. Telecommunications Press. 115-186.

[7] Zhang Haifeng \& Ai Ling. 2007. Study on nonlinear error elimination for DC Bridge output. Journal of Anyang Institute of Technology: 96-99.

[8] Zhang Lili. 2013. Principle and application of DC Bridge. Electronic science and technology.

[9] Zhang Mingjin. 2005. Analysis on measuring resistance by DC Bridge. China instrument: 114-116. 